Check for updates

Cite this: RSC Adv., 2017, 7, 46101

\title{
The effect of defects on the interfacial mechanical properties of graphene/epoxy composites
}

\author{
Maoyuan Li, ${ }^{a}$ Helezi Zhou, ${ }^{a}$ Yun Zhang, (D) *a Yonggui Liao ${ }^{b}$ and Huamin Zhou ${ }^{a}$
}

In this study, the effect of defects on the interfacial mechanical properties of graphene/epoxy composites has been investigated by using molecular dynamics simulations. Three common types of defects with different concentrations, i.e., single-vacancy, double-vacancy, and Stone-Wales, were considered. Two typical separations, i.e., normal separation and shear separation, were conducted to evaluate the graphene/epoxy interfacial cohesive strength and interfacial shear strength, respectively. The pull-out energy and corresponding forces during the whole process were determined. It was found that the vacancy defects, including single-vacancy and double-vacancy, would degrade the interfacial mechanical properties. In contrast, the Stone-Wales defect could enhance the interfacial strength, especially the interfacial shear strength. Besides, the effect of graphene agglomeration on the graphene/ epoxy interfacial shear stress was also investigated. The results showed that the interaction between graphene sheets was much stronger than that between graphene and polymer. Additionally, the graphene agglomerated structure was found to weaken the graphene sheet and epoxy interfacial load transfer, which agreed with the observations in previous experimental results in the literature.

Received 26th July 2017

Accepted 22nd September 2017

DOI: $10.1039 / c 7 r a 08243 f$

rsc.li/rsc-advances mechanical properties between Gr and polymer. For example, Lv et $a .^{10}$ evaluated that the interfacial bonding properties could be improved with the chemical functionalization of $\mathrm{Gr}$ when pulling the Gr out from the polymer matrix. Ding et al. ${ }^{11}$ found that the interfacial shear strength between graphene oxide and polymer was much stronger than that between $\mathrm{Gr}$ and polymer. Liu et al. ${ }^{12}$ showed that Gr grafted with polymer chains could improve the shear strength while degrade the cohesive strength.

However, most of the existing researches focused on the Gr without any defect. The defects ${ }^{\mathbf{1 3}}$ in the Gr sheets such as singlevacancy (SV), double-vacancy (DV) and Stone-Wales (SW) are inevitable during the synthesis process as well as manufacturing. Many experimental and simulation studies ${ }^{\mathbf{1 4 - 1 8}}$ have showed that the defects have a remarkable impact on the mechanical properties as well as other physical properties of the pristine Gr sheet. Moreover, some researches have been conducted to evaluate the effect of defects on the shear strength between carbon nanotube (CNT) and polymer. For example, Yang et al. ${ }^{19,20}$ showed that the vacancy defect would degrade the shear strength between CNT and polyethylene while the SW would enhance it, which was attributed to the stronger absorbed energy between the SW defective $\mathrm{Gr}$ and polymer. Similar results ${ }^{21}$ were obtained for CNT-epoxy nanocomposites by the introduction of SW defect and adatoms. More recently, Yang et $a .^{22}$ have firstly observed that the defect controlled by the high energy carbon ions could improve the shear strength of CNT/polymer derived ceramic interface while the measured fracture strength was observed to decrease marginally.

\footnotetext{
${ }^{a}$ State Key Laboratory of Material Processing and Die \& Mold Technology, Huazhong University of Science and Technology, Wuhan 430074, Hubei, China. E-mail: marblezy@hust.edu.cn; Tel: +86-27-87543492

${ }^{b}$ Key Laboratory for Large-Format Battery Materials and System, Ministry of Education, School of Chemistry and Chemical Engineering, Huazhong University of Science and Technology, Wuhan 430074, Hubei, China
}

Some studies by using MD method have proved that the chemical functionalization has influence on the interfacial 
Although there have been some pioneer simulation works as described above, there is few investigations on the effect of the defects on interfacial mechanical properties of Gr/epoxy nanocomposites, especially on the cohesive strength and shear strength. The mechanisms of enhancement or degradation effect on the interfacial mechanical properties caused by the $\mathrm{Gr}$ defects is still not well understood. Thus, it is of great significance to investigate the effect of defects on the interfacial mechanical properties of Gr/epoxy composites.

Besides, the agglomeration of $\mathrm{Gr}$ due to the lager surface area was another obstacle to improve the mechanical properties of polymer-based composites. To understand the degrade effect caused by $\mathrm{Gr}$ agglomeration was also a critical issue. Using MD simulations, Rahman et $a .^{23}$ found that the in-plane elastic modulus of well dispersed $\mathrm{Gr} /$ polymer nanocomposites was higher than that of the agglomerated one. Ding et al. ${ }^{11}$ also has showed that a well dispersion state of graphene oxide sheets could enhance the interfacial properties between the polymer and graphene oxide. However, little work has been reported todate on the effect of $\mathrm{Gr}$ agglomeration on interfacial mechanical properties. Additionally, the agglomeration of $\mathrm{Gr}$ may form more complex interface types in the Gr/polymer composites. Therefore, it is also necessary to investigate the effect of $\mathrm{Gr}$ agglomeration on the interfacial mechanical properties between Gr and epoxy considering the different interface types.

In this study, we have conducted a series of pull-out MD simulations to investigate the effect of defects on the interfacial mechanical properties of Gr-epoxy nanocomposites. Three common types of defects, i.e., SV, DV and SW were randomly dispersed in Gr sheets, respectively. Two separation modes i.e., the normal separation and the shear separation, were conducted to evaluate the cohesive strength and shear strength, respectively. Moreover, the effect of Gr agglomeration on the shear strength was also studied by different pull-out manners.

\section{Computational methods}

\subsection{Molecular models of Gr/epoxy nanocomposites}

The molecular models of $\mathrm{Gr} / \mathrm{epoxy}$ nanocomposites were first constructed by a commercial software, Material Studio (Accelrys). A representative molecular method proposed by $\mathrm{Yu}$ et al. $^{24}$ was used to build the initial cross-linked epoxy model. In this model, six molecular chains of bisphenol A diglycidyl ether (DGEBA) resin were linked by one molecular chain of triethylenetetramine (TETA) curing agent, as shown in Fig. 1. The method has been used to successfully obtain the thermal and mechanical properties ${ }^{23,25-27}$ of cross-linked epoxy, and the simulation results agreed well with experimental observations.

To evaluate the interfacial properties of $\mathrm{Gr} / \mathrm{epoxy}$ composites, two representative interface models were conducted. One is the normal separation, which was constructed to obtain the cohesive strength by applying $z$-axis displacement to the $\mathrm{Gr}$, as shown in Fig. 2a. Another is the shear separation, which was conducted to obtain the shear strength by applying $x$-axis displacement to pull out the Gr from epoxy matrix, as shown in Fig. 2b. The molecular model of cross-linked epoxy and pristine Gr sheet were shown in Fig. 2c and d. The molecular

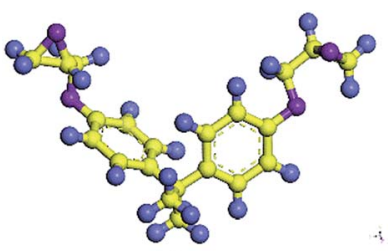

(a)

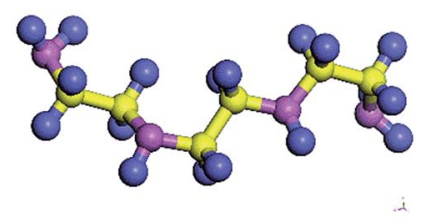

(b)
Fig. 1 Molecular model of (a) epoxy resin (DGEBA), (b) curing agent (TETA) (blue balls are hydrogen atoms, purple balls are oxygen atoms, lilac balls are nitrogen atoms, yellow balls are carbon atoms in epoxy, respectively).

configurations of the above models were listed in Table 1 . To investigate the effect of defects on the interfacial mechanical properties of Gr/epoxy nanocomposites, Gr sheets with three common types of possible defects, including SV, DV, and SW were constructed, as shown in Fig. 3 . The SV and DV defects were formed by removing one carbon atom and two adjacent carbon atoms on the pristine Gr sheet, respectively. The SW defect was created by rotating one of the C-C bonds by $90^{\circ}$, transforming four hexagons into two pentagons and heptagons (5-7-7-5). The concentration of defects ranged from $0 \%$ to $13 \%$, and the three types of defects were randomly located on the $\mathrm{Gr}$ sheets, respectively. For the vacancy defect, the concentration was defined as the number density of atoms removed from the Gr sheet. For a SW defect, which did not involve any removed or added carbon atoms in Gr sheet, the concentration was defined by considering two defected atoms.

In the MD simulations, an ab initio force field, polymer consistent force field (PCFF ${ }^{28}$ based on CFF91 with additional parameters was used to describe all the inter- and intra-atomic interactions for the nanocomposites. This force field has been widely used in previous studies to investigate the mechanical and thermal properties of $\mathrm{Gr}$ reinforced epoxy composites. ${ }^{5,29,30}$

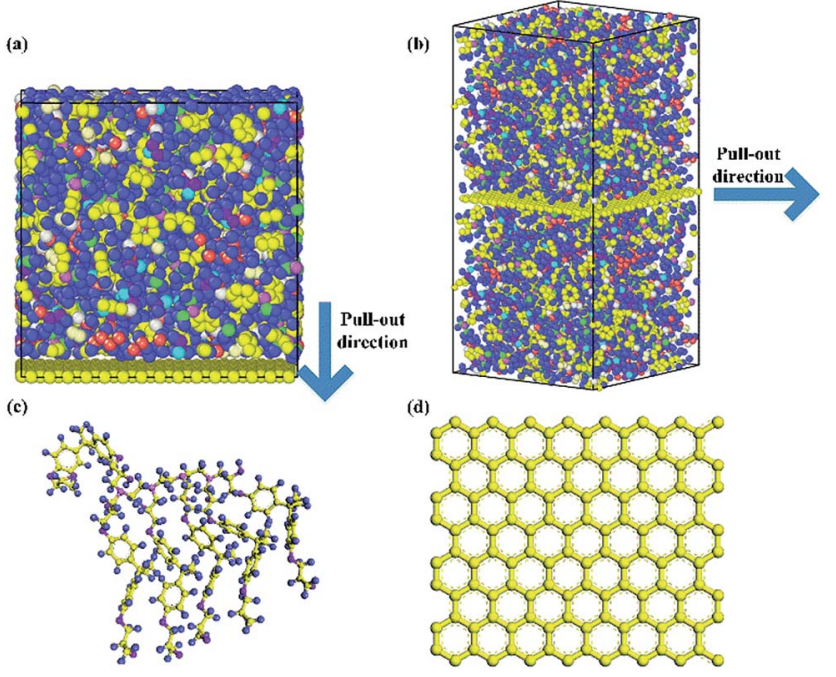

Fig. 2 Gr/epoxy nanocomposites of (a) normal separation interface model and (b) shear separation interface model; (c) representative molecule DEGBA cured with TETA; (d) pristine Gr sheet. 
Table 1 Material configuration of the normal separation and the shear separation interface model

\begin{tabular}{llll}
\hline Configuration & $\begin{array}{l}\text { Unit cell } \\
\text { dimension }(\AA)\end{array}$ & Atom number & $\begin{array}{l}\text { Interaction } \\
\text { force type }\end{array}$ \\
\hline $\begin{array}{l}\text { Normal separation } \\
\text { interface model }\end{array}$ & $\begin{array}{l}a=36.4 b=37.14 \\
c=39.22\end{array}$ & 5370 & Normal \\
$\begin{array}{l}\text { Shear separation } \\
\text { interface model }\end{array}$ & $\begin{array}{l}a=36.1 b=37.5 \\
c=73.13\end{array}$ & 10200 & Shear \\
\end{tabular}

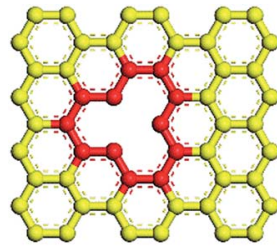

(a)

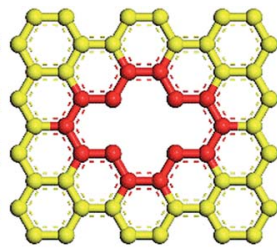

(b)

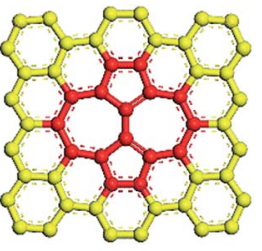

(c)
Fig. 3 Types of defects studied in this work (a) single-vacancy (SV), (b) double-vacancy (DV) and (c) Stone-Wales (SW) defect.

Assuming there is no covalent chemical bonding between $\mathrm{Gr}$ and epoxy, the interfacial bonding strength was mainly dominated by van der Waals (vdW) interaction, which was expressed as the 9-6 Lennard-Jones (LJ) potentials in PCFF. The 9-6 LJ potential is described as $E_{i j}=\varepsilon\left[2\left(\delta / r_{i j}\right)^{9}-3\left(\delta / r_{i j}\right)^{6}\right]$, where $r_{i j}$ is the distance between atom $i$ and $j, \varepsilon$ and $\delta$ are the energy and distance constants, respectively. The LJ potential parameters between different types of atoms are calculated by using the sixth power mix rules (i.e., $\varepsilon_{i j}=\left(2 \sqrt{\varepsilon_{i} \varepsilon_{j}} \delta_{i}{ }^{3} \delta_{j}{ }^{3}\right) /\left(\delta_{i}{ }^{6}+\delta_{j}{ }^{6}\right) ; \delta_{i j}=$ $\left.\left(\left(\delta_{i}{ }^{6}+\delta_{j}{ }^{6}\right) / 2\right)^{1 / 6}\right)$. The cutoff distance for the calculation of van der Waals (vdW) interactions was $1.0 \mathrm{~nm}$ in the present simulations. The Large-scale Atomic/Molecular Massively Parallel Simulator (LAMMPS) ${ }^{31}$ was used to conduct all the molecular dynamic simulation, and the velocity-Verlet method was used to integrate the equation of motion. The main steps involved in the pull-out simulations for composites containing Gr sheet with or without defects are as follows:

2.1.1 Step 1-equilibration. At the beginning, total potential energy of the composite system was minimized by using a conjugate gradient algorithm. The system was then relaxed in a canonical NVT ensemble (i.e., constant number of atoms, volume and temperature) for $100 \mathrm{ps}(\Delta t=0.25 \mathrm{fs})$, during which the temperature was increased from $1 \mathrm{~K}$ to $300 \mathrm{~K}$ at a rate of $3 \mathrm{~K} \mathrm{ps}^{-1}$, kept at $300 \mathrm{~K}$ for $50 \mathrm{ps}(\Delta t=0.25 \mathrm{fs})$, and then cooled down to $1 \mathrm{~K}$ at the same rate. Subsequently, the system was relaxed in an isothermal-isobaric NPT ensemble (i.e., constant number of atoms, pressure and temperature) at $1 \mathrm{~K}$ and $1 \mathrm{~atm}$ for $100 \mathrm{ps}$ ( $\Delta t=0.25 \mathrm{fs}$ ). The purpose of this step was to achieve a stress-free state. Thereafter, the system was finally relaxed in the NVT ensemble for a further $100 \mathrm{ps}(\Delta t=0.25 \mathrm{fs})$ at a temperature of $1 \mathrm{~K}$.

2.1.2 Step 2-pull-out simulation. Followed by the equilibration, the pull-out simulations were conducted to determine the cohesive strength and shear strength of the pristine (defective) Gr/epoxy interface. The Gr sheet was pulled out at a velocity of $0.0001 \AA \mathrm{fs}^{-1}$ until it was completely pulled out from the epoxy matrix. Followed by previous studies, ${ }^{29,32}$ the $\mathrm{Gr}$ sheet was treated as a rigid body during the pull-out process, the reason for the treatment was that the stiffness of Gr sheet is much higher than that of polymer matrix, there is no obvious temperature variation and the pull-out velocity was comparatively fast in the MD simulations. The periodic boundary conditions (PBCs) were applied in the all directions, except the $x / z$-axis, i.e., the pull-out direction of $\mathrm{Gr}$ sheets.

\subsection{The calculation of interfacial properties}

The interfacial interaction mechanical properties between $\mathrm{Gr}$ and epoxy matrix can be evaluated by the interfacial energy $\Delta E$, pull-out energy $E_{\text {pullout }}$ and pull-out force $F_{\text {pullout }}$. The interaction energy was calculated from the different between the potential energy of the nanocomposite and the potential energy of epoxy matrix and the Gr filler, as follow: ${ }^{\mathbf{3 3 , 3 4}}$

$$
\Delta E=E_{\text {total }}-E_{\mathrm{Gr}}-E_{\text {epoxy }}
$$

where $E_{\text {total }}$ was the total energy of the composites, $E_{\mathrm{Gr}}$ and $E_{\text {epoxy }}$ denoted the potential energy of Gr and epoxy, respectively. Since the pull-out energy is the work required by applying a shear force to pull the graphene out from the polymer matrix, the interfacial mechanical properties, i.e., interfacial shear strength can be obtained from the pull-out energy. The most of the carbon atoms in the graphene are electrically neutral and no covalent bonds are formed between graphene and polymer matrix, the interaction energy between graphene and matrix was mainly attributed to the vdW interaction, thus, the pull-out energy $E_{\text {pullout }}$ can be estimated from the potential energy difference between the initial and complete pull-out state: $\mathbf{3 0}^{\mathbf{3 0 , 3 4 , 3 5}}$

$$
E_{\text {pullout }}=E_{\text {pullout }}^{\text {current }}-E_{\text {pullout }}^{\text {inital }}
$$

where the superscripts "current" and "initial" respectively indicated the current pull-out state and the state before the pullout simulation started. The pull-out force $F_{\text {pullout }}$ was obtained by summing up the pull-out direction component of forces on all the atoms in the $\mathrm{Gr}^{32}$

For normal separation, the cohesive strength $\sigma_{i}$ can be calculated from the following equation:

$$
\sigma_{i}=\frac{F_{z-\max }}{w L}
$$

where $\sigma_{i}$ is the cohesive strength, $F_{\mathrm{z}-\mathrm{max}}$ is the maximum pullout force in the separation direction, $w, L$ are the width and length of the $\mathrm{Gr}$, respectively.

For shear separation, the shear strength $\tau_{i}$ can be calculated from the pull-out energy as follows:

$$
\begin{gathered}
E_{\text {pullout }}=\int_{0}^{L} 2 w(L-x) \tau_{i} \mathrm{~d} x=w \tau_{i} L^{2} \\
\tau_{i}=\frac{E_{\text {pullout }}}{w L^{2}}
\end{gathered}
$$


where $w, L$ are the width and length of the Gr, respectively. $x$ was the distance of Gr during the pullout process. The values $\tau_{i}$ were obtained from three independent simulations on the same condition, and the final value was the average of all calculated values.

\section{Results and discussion}

\subsection{Validation of MD model}

To validate the molecular models and PCFF potential, the cohesive strength and shear strength between the pristine $\mathrm{Gr}$ and cross-linked epoxy were first calculated. Fig. 4 shows the snapshots of the normal/shear separation pull-out process of the pristine Gr from the epoxy matrix, respectively. The pull-out force $F_{\text {pullout }}$ and pull-out energy $E_{\text {pullout }}$ during the pullout process were calculated, as shown in Fig. 5. For the case of normal separation, the pull-out force increased linearly at first, and reached a maximum of about $180 \mathrm{kcal} \mathrm{mol}^{-1} \AA^{-1}$ at around $1 \AA$ A separation, beyond which it reduced gradually and went to zero, which agreed with previous studies. ${ }^{12,36}$ For the case of shear separation, it can be observed that the pull-out force can be divided into three stages during the whole process. The pullout force increased in the first stage, then oscillated around a stable value in the second stage, and finally decreased in the third stage, which also had the same trend with previous studies. ${ }^{23,37}$ The length of the first and the third stage was about $1.0 \mathrm{~nm}$, which was close to the cutoff distance of vdW interaction. The pull-out energy $E_{\text {pullout }}$ for the two separation ways increased gradually as the Gr was pulled out from the epoxy matrix. The variation of energy during the whole pull-out process was found to be equal to the variation of the vdW energy, which indicated the interfacial bonding strength was mainly dominated by vdW interaction.

Based on the calculated pull-out force, pull-out energy and eqn (2)-(5), the cohesive strength and shear strength between the pristine $\mathrm{Gr}$ and epoxy were 962.77 $\mathrm{MPa}$ and 111.10 MPa, respectively. The results showed that the interfacial cohesive strength was much higher than the interfacial shear strength, implying that the shear separation failure may be generated more easily compared with the normal separation. The value of cohesive strength in present study agreed with the simulation results by other researchers ${ }^{12,29,36}$ (i.e., $\sim 730 \mathrm{MPa}$ ). The difference was due to the different polymer matrix type and the unit cell sizes. Besides, the cohesive strength (i.e., 962.77 MPa) between $\mathrm{Gr}$ and polymer was much larger than that (i.e., 479 $\mathrm{MPa}$ ) between carbon nanotube (CNT) and polymer, ${ }^{38}$ indicating that the Gr had a better enhancement effect than CNT in the polymer nanocomposites. ${ }^{1}$ For the case of shear separation, the calculated shear strength for the present model was also consistent with the previous studies ${ }^{\mathbf{1 0 , 3 2 , 3 9}}$ (i.e., $\sim 100 \mathrm{MPa}$ ). Additionally, the results indicated that the interfacial shear strength between Gr and epoxy was much larger than that of CNT/epoxy (40.68 MPa) ${ }^{40}$ In the later discussion, these cohesive strength and shear strength values between pristine $\mathrm{Gr}$ and epoxy will be used as the reference. Hence, these results confirmed the validity of the PCFF potential and present molecular models to calculate the interfacial bonding strength between $\mathrm{Gr}$ and polymer.

\subsection{Effect of defects on the interfacial properties}

The effect of the three kinds of defects on the interfacial properties of Gr/epoxy are discussed in this section. As described in the previous section, both the normal separation and shear separation were considered. For the case of normal separation mode, the cohesive strength between Gr-epoxy and the maximum force in $z$-axis required to separate the Gr from epoxy are calculated according to the concentrations of different defect type, as shown in Fig. 6. In case of the vacancy defects, including $\mathrm{SV}$ and $\mathrm{DV}$, the results revealed that both the maximum force in pullout direction and the corresponding cohesive strength of the Gr-epoxy decreased as the defect concentrations in the Gr sheet increased. Since the bonded energy of the epoxy and Gr didn't change remarkably, the non- (a)

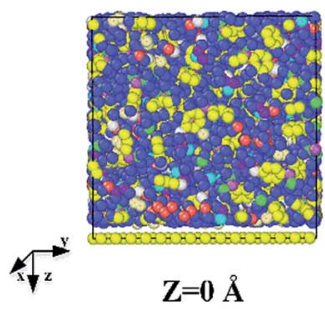

(b)

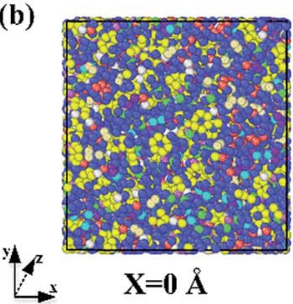

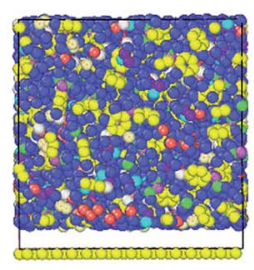

$\mathbf{Z}=\mathbf{2} \AA$

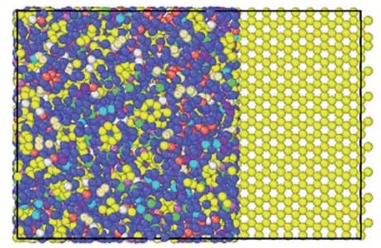

$\mathbf{X}=\mathbf{2 0} \AA$

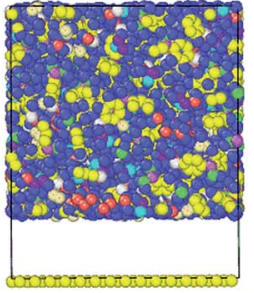

$\mathrm{Z}=\mathbf{8} \AA$

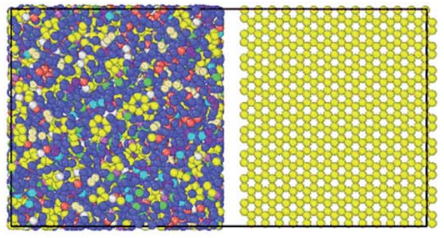

$\mathrm{X}=36 \AA$

Fig. 4 Snapshots of the pull-out process for (a) normal separation mode (b) shear separation mode. 


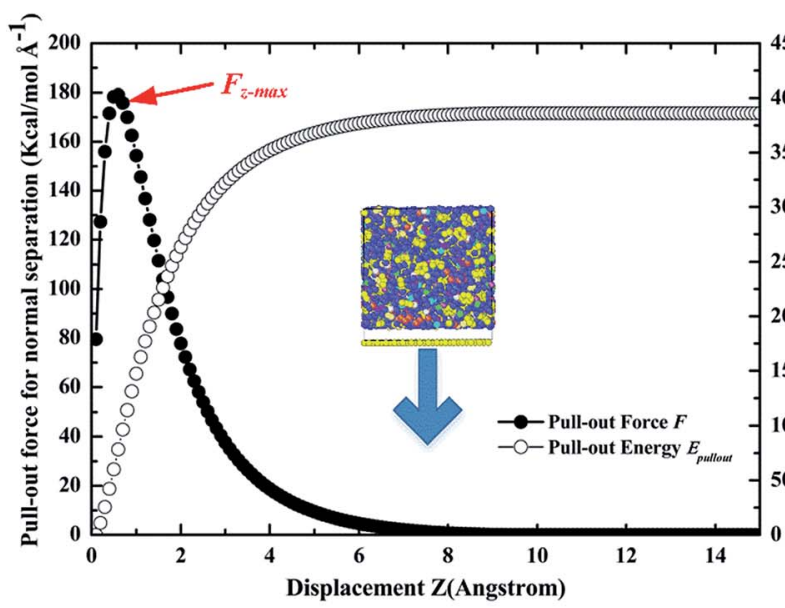

(a)

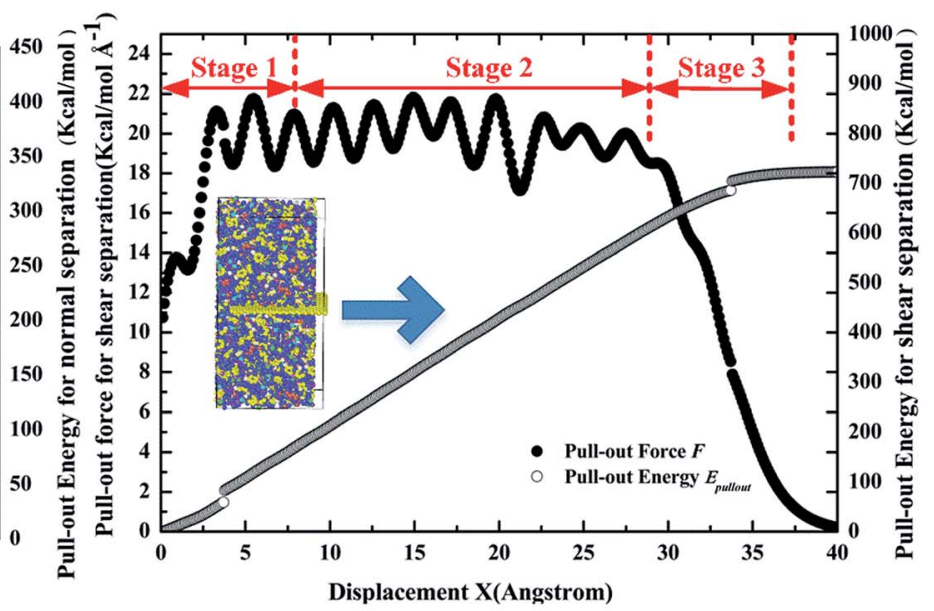

(b)

Fig. 5 Variation of the pull-out force $F$ and pull-out energy $E_{\text {pullout }}$ for the nanocomposite containing pristine Gr during the pull-out process (a) normal separation mode (b) shear separation mode.

bonded energy, that is, the vdW interaction between the Gr and epoxy matrix completely contributed to the interaction energy. According to the expressions, the vdW interaction energy would decrease as the increased number of removed carbon atoms in the $\mathrm{Gr}$, resulting in the decrease of the cohesive strength. Unlike the vacancy defect, the existence of SW defect enhanced the cohesive strength and the maximum pullout force. It can be observed that the cohesive strength increased from 962.77 to the 991.16 MPa when the SW defect concentration was 3\%. The enhancement effect in the cohesive strength indicated that the interaction energy between SW defective Gr and epoxy was stronger than that between pristine $\mathrm{Gr}$ and epoxy.

For the case of shear separation mode, the similar results had been found that the vacancy defects (SV and DV defect) could degrade the shear strength while the SW defect had a reinforcement effect on the shear strength and the corresponding pull-out energy, as shown in Fig. 7. Comparing to the cohesive strength, the shear strength showed a more sensitive relationship with the vacancy defect, for example, the maximum decrease in the shear strength was about 31\% (111.10 to 76.61 $\mathrm{MPa})$ while the result in cohesive strength was about $17 \%(962.77$ to $821.73 \mathrm{MPa}$ ) at SV defect with a concentration of $13 \%$. Furthermore, the SV defect had a more significant decrease effect on shear strength and the pullout energy than the DV defect at the same defect concentrations, for instance, the shear strength decreased to 102.31, 93.65 MPa for a defect concentration of $8 \%$ of SV and DV, respectively. Previous simulations have indicated that the energy per missing atom for a DV was much lower than that for $\mathrm{SV},^{13}$ thus, the DV defect was thermodynamically preferred than SV defect. The SW defect showed a positive effect on the shear strength, of which the maximum increase was about $4 \%$ at a concentration of $5 \%$. Meanwhile, the corresponding pullout energy increased with the existence of SW, indicating a stronger adhesive effect compared with the pristine Gr.

The above results agreed with some previous works. ${ }^{19-22,41}$ However, the mechanism of the enhancement in shear strength

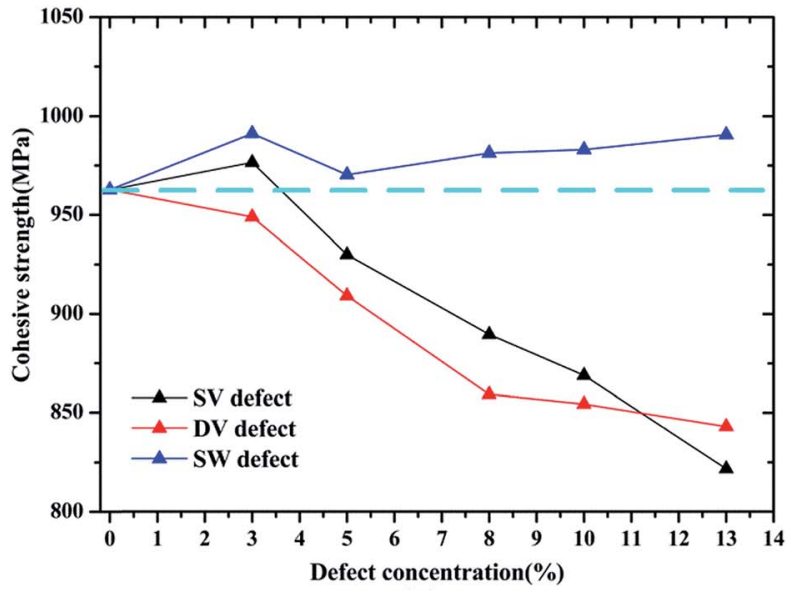

(a)

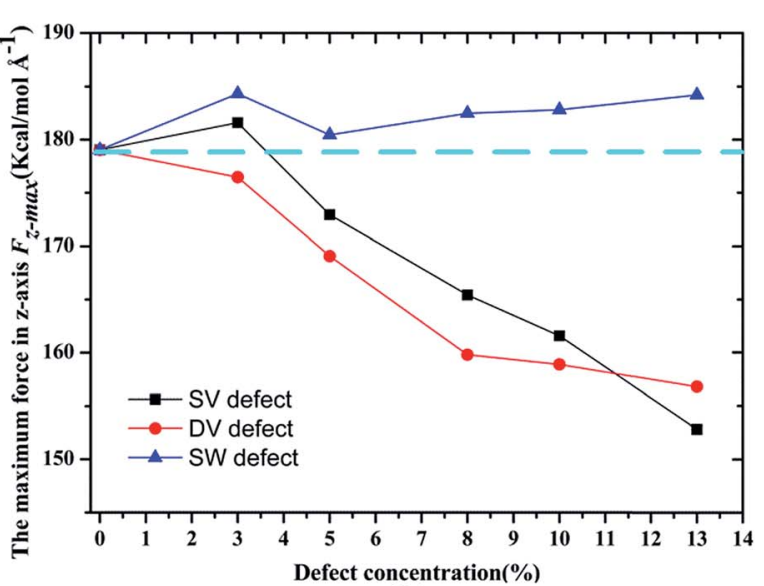

(b)

Fig. 6 Variation in (a) cohesive strength $\sigma_{i}$ and (b) the maximum force $F_{z-\max }$ in $z$-axis according to concentrations of different defect types. 
and cohesive strength by SW defect was complex, as the formation of SW defect didn't remove or add any atoms. Yang et $a{ }^{19,20}$ concluded from their studies that the enhancement may be attributed to the fact that the polymer molecules were more efficiently absorbed on the surface of the SW defective Gr. Some other studies ${ }^{21}$ found that the SW defects can increase the concentration of $\mathrm{sp}^{2}$ carbons near the Gr, leading to the increase in $\pi-\pi$ attractions at the Gr-epoxy interface. More recently, Moon et al. ${ }^{\mathbf{4 1}}$ have revealed the enhancement mechanism between the SW defective Gr and polymer through a density functional theory (DFT) simulation, an improved adsorption energy caused by SW defect was clearly observed using the quantum simulations. Moreover, the SW defects were also found to increase the sliding resistance of polymer on $\mathrm{Gr}$ sheet, leading to more polymer molecules absorbed onto the Gr. It should be noted that the cohesive strength and shear strength were evaluated using eqn (3)-(5), however, the Gr surface area may be changed with the introduction of defect, for example, the Gr sheet would form the wrinkled structure caused by the SW defect, leading to the increase or decrease of the surface area. Thus, it is difficult to calculate the wrinkled Gr area accurately. ${ }^{32}$ All in all, the above results indicate that the vacancy defect, including SV and DV, could degrade the interfacial properties of Gr-epoxy, and the SW defect could enhance the cohesive strength and shear strength in Gr-epoxy system. Therefore, the introduction of SW defect in Gr sheets may be a useful way to improve the mechanical strength of $\mathrm{Gr} /$ polymer nanocomposites.

\subsection{Effect of agglomeration on the interfacial property}

Based on the preceding study on the Gr-epoxy interfacial properties, we continued evaluating the effect of agglomeration on the interfacial property, which was commonly observed in fabricating the $\mathrm{Gr}$ /epoxy nanocomposites. Followed by previous study, ${ }^{11,23}$ a three-layer Gr/epoxy composites molecular model was conducted to investigate the influence of Gr agglomerations. The shear separation was applied in this part.
For the three-layer Gr, different kinds of pull-out manners may happen under the practical circumstance. Thus, three typical manners were studied, i.e., pulling out the whole $\mathrm{Gr}$ sheets (Case 2), pulling out the middle Gr sheet (Case 3), and pulling out the top Gr sheet (Case 4). The Case 1, i.e., pulling out the single Gr sheet, was also studied as the reference. The pullout force and energy during the whole process for the four cases were calculated, as shown in Fig. 8. Compared with the singleGr system (Case 1), it can be observed that the pull-out energy increased when more Gr sheets (Case 2) were pulled out. For the triple-Gr system, the order of the pull-out energy for different cases was: Case $3>$ Case $4>$ Case 2 . The pull-out energy mainly originated from the interaction energy (vdW interactions) between the top/bottom epoxy matrix and the whole Gr sheets (Case 2), the top-bottom Gr sheets and the middle Gr sheet (Case 3), the upper Gr sheet/below epoxy matrix and the bottom Gr sheet (Case 4), respectively. The above results indicated that the interaction energy between Gr sheets was stronger than that between Gr and epoxy. A similar simulation result was also found in recent study of the Gr/PVA nanocomposites system. ${ }^{\mathbf{1 1}}$ Meanwhile, the corresponding pull-out forces were also calculated and found to have the same trend with the pull-out energy, as shown in the inset in Fig. 9.
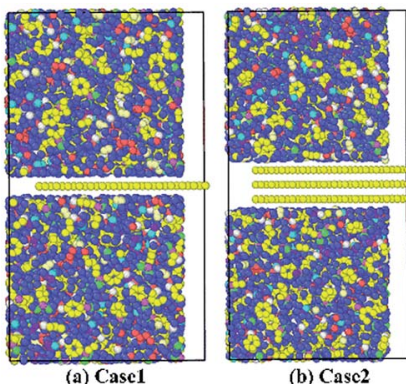

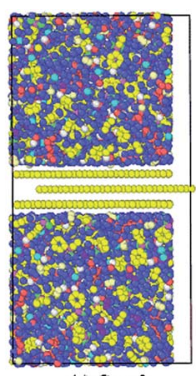

(c) Case 3

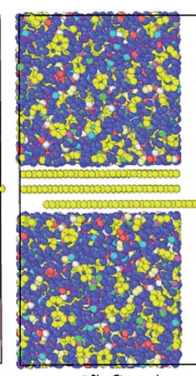

(d) Case 4
Fig. 8 Pulling out (a) the single Gr sheet; (b) the whole Gr sheets; (c) the middle Gr sheet; (d) the bottom Gr sheet.

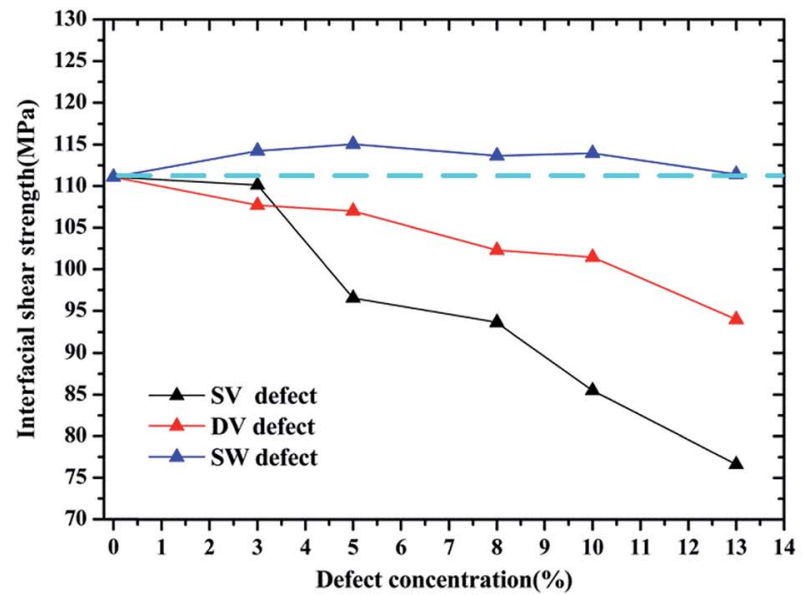

(a)

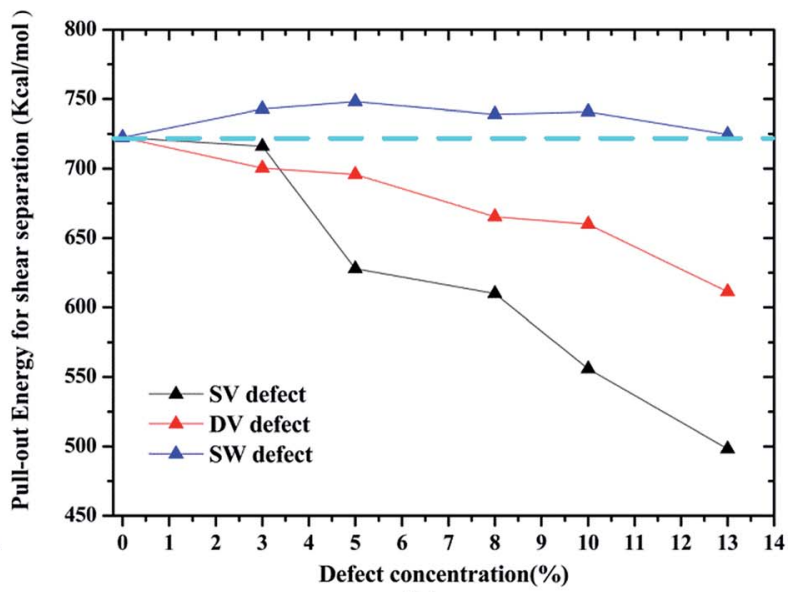

(b)

Fig. 7 Variation in (a) interfacial shear strength $\tau_{i}$ and (b) the pull-out energy $E_{\text {pullout }}$ according to concentrations of different defect types. 


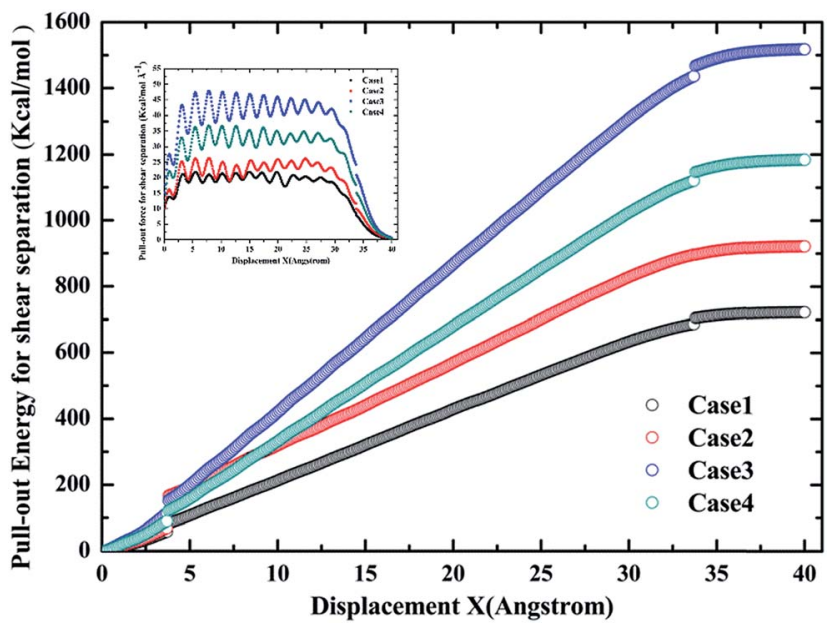

Fig. 9 The pull-out energy profiles for different cases during the whole process. The inset shows the corresponding pull-out force during the whole process.

The values of contact areas, pullout energy and the shear strength for different Gr/epoxy nanocomposite systems were presented in Table 2. Using the eqn (4) and (5), the calculated shear strength for Case 1 to 4 was 111.11, 105.37, 233.24 and 181.92 $\mathrm{MPa}$, respectively. It should be noted that, for the Case 2 pullout process of triple-Gr systems, the shear strength, $\tau_{i}$, can be calculated by the follow expressions:

$$
\begin{gathered}
E_{\text {pullout }}=\int_{0}^{L} 2(w+h)(L-x) \tau_{i} \mathrm{~d} x=(w+h) \tau_{i} L^{2} \\
\tau_{i}=\frac{E_{\text {pullout }}}{(w+h) L^{2}}
\end{gathered}
$$

where $w, L$ are the width and length of the Gr, respectively. $h$ was the height of the $\mathrm{Gr}$ sheet, i.e., $0.34 \mathrm{~nm}$ was the distance between two sheets. $x$ was the distance of Gr during the pullout process. Unlike the pullout energy and force, the shear strength of the triple-Gr (Case 2) was weaker than the single-Gr system (Case 1), indicating that the single-Gr could provide a better load transfer, that is, the agglomeration of Gr structure may weaken the interfacial performance of Gr-epoxy. Comparing the values of the shear strength of Case 2 to 4 , it can be found that

Table 2 Pull-out energy and shear strength for different Gr/epoxy nanocomposite systems

\begin{tabular}{llll}
\hline $\begin{array}{l}\text { Nanocomposites } \\
\text { system }\end{array}$ & $\begin{array}{l}\text { Contact area, } \\
L^{2}(w+h)\left(\AA^{3}\right)\end{array}$ & $\begin{array}{l}E_{\text {pull-out }} \\
\left(\mathrm{kcal} \mathrm{mol}^{-1}\right)\end{array}$ & $\begin{array}{l}\text { Interfacial } \\
\text { shear strength, } \\
\tau_{i}(\mathrm{MPa})\end{array}$ \\
\hline $\begin{array}{l}\text { Single-Gr } \\
(\text { Case 1) }\end{array}$ & 45116.85 & 722.52 & 111.11 \\
$\begin{array}{l}\text { Triple-Gr } \\
(\text { whole, Case 2) }\end{array}$ & 57589.16 & 874.64 & 105.37 \\
$\begin{array}{l}\text { Triple-Gr } \\
\text { (middle, Case 3) }\end{array}$ & 45116.85 & 1517.73 & 233.24 \\
$\begin{array}{l}\text { Triple-Gr } \\
\text { (bottom, Case 4) }\end{array}$ & 45116.85 & 1183.97 & 181.92
\end{tabular}

the interfacial strength between Gr sheets was stronger than that between the Gr and epoxy. In other words, it was easier to pullout the whole Gr sheets from the epoxy matrix than pulling out the middle Gr sheet from the multilayer Gr systems. This also can be evaluated by our recent experimental study on the Gr/epoxy fracture, pulling out of single Gr sheet from epoxy matrix was hard to find on the fracture surface morphology. ${ }^{4}$ The above results could provide an explanation for some experimental results ${ }^{42}$ that the agglomeration of Gr may limit the mechanical properties of $\mathrm{Gr}$ based nanocomposites by experiment as the theory expected.

\section{Conclusion}

The effect of defects on the interfacial mechanical properties of Gr/epoxy nanocomposites were investigated by MD simulations in present study. Three common types of defects were studied, including SV, DV and SW. To determine the interfacial mechanical properties, two modes, i.e., the normal separation and the shear separation, were studied. Among the three defects, the vacancy defects, including SV and DV, were found to degrade both the cohesive strength and shear strength as the defect concentration increased from $0 \%$ to $13 \%$, which were due to the vdW interaction energy decreased with the decreasing number of carbon atoms. The shear strength showed a more sensitive relationship with the defects compared with the cohesive strength, for instance, the maximum decrease in the shear strength was about $31 \%$ (111.10 to $76.61 \mathrm{MPa})$ while the result in cohesive strength was about $17 \%$ (962.77 to 821.73 $\mathrm{MPa}$ ) for SV defect with a concentration of $13 \%$. Moreover, the SV was found to degrade the shear strength more seriously compared with the DV at the same defect concentration. On the contrary, the introduction of SW defect was found to enhance the cohesive strength and shear strength, with a maximum increase of about $3 \%$ and $4 \%$, respectively. The underlying enhancement mechanism can be explained by the fact that the absorbed energy between the SW defective Gr and epoxy was stronger than that for the pristine Gr. In addition, the existence of SW would increase in $\pi-\pi$ attractions at the Gr-epoxy interface, leading to a better interfacial load transfer in $\mathrm{Gr}-$ epoxy system. The above results indicate that introduction of moderate SW defects in Gr may be a useful method to improve the mechanical performance of Gr/polymer nanocomposites.

Besides, the effect of agglomeration on the shear strength was also investigated, the results showed that the interaction energy between Gr sheets was much stronger than that between Gr and epoxy. The agglomeration of Gr would degrade the shear strength between the Gr and epoxy compared with that when the Gr well dispersed in matrix, which agreed with the observation from the previous experimental results in the literature.

\section{Conflicts of interest}

The authors declare no competing financial interest. 


\section{Appendix 1}

\section{Abbreviations and acronyms}

$\begin{array}{ll}\text { Gr } & \text { Graphene } \\ \text { MD } & \text { Molecular dynamics } \\ \text { SV } & \text { Single-vacancy } \\ \text { DV } & \text { Double-vacancy } \\ \text { SW } & \text { Stone-Wales } \\ \text { DGEBA } & \text { Bisphenol A diglycidyl ether } \\ \text { TETA } & \text { Triethylenetetramine } \\ \text { CNT } & \text { Carbon nanotube } \\ \text { PCFF } & \text { Polymer consistent force field } \\ \text { vdW } & \text { van der Waals }\end{array}$

\section{Acknowledgements}

The authors would like to acknowledge financial support from the National Natural Science Foundation of China (Grant No. 51210004, 51635006, 51675199) and Fundamental Research Funds for the Central Universities (Grant No. 2016YXZD059, 2015ZDTD028).

\section{Notes and references}

1 M. A. Rafiee, J. Rafiee, Z. Wang, H. Song, Z.-Z. Yu and N. Koratkar, ACS Nano, 2009, 3, 3884-3890.

2 H. Kim, A. A. Abdala and C. W. Macosko, Macromolecules, 2010, 43, 6515-6530.

3 S. Stankovich, D. A. Dikin, G. H. B. Dommett, K. M. Kohlhaas, E. J. Zimney, E. A. Stach, R. D. Piner, S. T. Nguyen and R. S. Ruoff, Nature, 2006, 442, 282-286.

4 X. Du, H. Zhou, W. Sun, H.-Y. Liu, G. Zhou, H. Zhou and Y.-W. Mai, Compos. Sci. Technol., 2017, 140, 123-133.

5 F. Lin, Y. Xiang and H.-S. Shen, Composites, Part B, 2017, 111, 261-269.

6 C. Li, A. R. Browning, S. Christensen and A. Strachan, Composites, Part A, 2012, 43, 1293-1300.

7 L. Gong, I. A. Kinloch, R. J. Young, I. Riaz, R. Jalil and K. S. Novoselov, Adv. Mater., 2010, 22, 2694-2697.

8 S. Gong, H. Ni, L. Jiang and Q. Cheng, Mater. Today, 2017, 20, 210-219.

9 C.-Y. Chang, S.-P. Ju, J.-W. Chang, S.-C. Huang and H.-W. Yang, RSC Adv., 2014, 4, 26074-26080.

10 C. Lv, Q. Xue, D. Xia, M. Ma, J. Xie and H. Chen, J. Phys. Chem. C, 2010, 114, 6588-6594.

11 N. Ding, X. Chen, C.-M. L. Wu and X. Lu, J. Phys. Chem. C, 2012, 116, 22532-22538.

12 F. Liu, N. Hu, J. Zhang, S. Atobe, S. Weng, H. Ning, Y. Liu, L. Wu, Y. Zhao, F. Mo, S. Fu, C. Xu, Alamusi and W. Yuan, RSC Adv., 2016, 6, 66658-66664.

13 F. Banhart, J. Kotakoski and A. V. Krasheninnikov, ACS Nano, 2011, 5, 26-41.

14 Y. Ren and G. Cao, Carbon, 2016, 103, 125-133.
15 A. Zandiatashbar, G.-H. Lee, S. J. An, S. Lee, N. Mathew, M. Terrones, T. Hayashi, C. R. Picu, J. Hone and N. Koratkar, Nat. Commun., 2014, 5, 3186.

16 Y. Wei, J. Wu, H. Yin, X. Shi, R. Yang and M. Dresselhaus, Nat. Mater., 2012, 11, 759-763.

17 N. Jing, Q. Xue, C. Ling, M. Shan, T. Zhang, X. Zhou and Z. Jiao, RSC Adv., 2012, 2, 9124-9129.

18 G. Rajasekaran and A. Parashar, RSC Adv., 2016, 6, 2636126373.

19 S. Yang, S. Yu and M. Cho, Carbon, 2013, 55, 133-143.

20 S. Yang, J. Choi and M. Cho, Compos. Struct., 2015, 127, 108119.

21 X. Peng and S. A. Meguid, Comput. Mater. Sci., 2017, 126, 204-216.

22 Y. Yang, C. Ramirez, X. Wang, Z. Guo, A. Tokranov, R. Zhao, I. Szlufarska, J. Lou and B. W. Sheldon, Carbon, 2017, 115, 402-408.

23 R. Rahman and A. Haque, Composites, Part B, 2013, 54, 353364.

24 S. Yu, S. Yang and M. Cho, Polymer, 2009, 50, 945-952.

25 S.-C. Shiu and J.-L. Tsai, Composites, Part B, 2014, 56, 691697.

26 B. Kim, J. Choi, S. Yang, S. Yu and M. Cho, Polymer, 2015, 60, 186-197.

27 H. Shin, S. Yang, S. Chang, S. Yu and M. Cho, Polymer, 2013, 54, 1543-1554.

28 H. Sun, J. Phys. Chem. B, 1998, 102, 7338-7364.

29 F. Liu, N. Hu, H. Ning, S. Atobe, C. Yan, Y. Liu, L. Wu, X. Liu, S. Fu, C. Xu, Y. Li, J. Zhang, Y. Wang and W. Li, Carbon, 2017, 115, 694-700.

30 Q. L. Xiong, S. A. Meguid, Y. Wang and G. J. Weng, Mech. Mater., 2015, 85, 38-46.

31 S. Plimpton, J. Comput. Phys., 1995, 117, 1-19.

32 F. Liu, N. Hu, H. Ning, Y. Liu, Y. Li and L. Wu, Comput. Mater. Sci., 2015, 108, 160-167.

33 J. Gou, B. Minaie, B. Wang, Z. Liang and C. Zhang, Comput. Mater. Sci., 2004, 31, 225-236.

34 Q. Zheng, Q. Xue, K. Yan, X. Gao, Q. Li and L. Hao, Polymer, 2008, 49, 800-808.

35 Q. Zheng, D. Xia, Q. Xue, K. Yan, X. Gao and Q. Li, Appl. Surf. Sci., 2009, 255, 3534-3543.

36 P. A. Amnaya, C. L. Dimitris and C. H. Daniel, Modell. Simul. Mater. Sci. Eng., 2009, 17, 015002.

37 M. C. Wang, Z. B. Lai, D. Galpaya, C. Yan, N. Hu and L. M. Zhou, J. Appl. Phys., 2014, 115, 123520.

38 H. Tan, L. Y. Jiang, Y. Huang, B. Liu and K. C. Hwang, Compos. Sci. Technol., 2007, 67, 2941-2946.

39 C. Lv, Q. Xue, D. Xia and M. Ma, Appl. Surf. Sci., 2012, 258, 2077-2082.

40 Q. L. Xiong and S. A. Meguid, Eur. Polym. J., 2015, 69, 1-15. 41 J. Moon, S. Yang and M. Cho, Carbon, 2017, 118, 66-77.

42 L.-C. Tang, Y.-J. Wan, D. Yan, Y.-B. Pei, L. Zhao, Y.-B. Li, L.-B. Wu, J.-X. Jiang and G.-Q. Lai, Carbon, 2013, 60, 16-27. 